\title{
Cold Atmospheric Plasma Treatment of Medical Devices
}

\author{
Kotov Dmitry ${ }^{1}$, Krivenchuk Dmitry ${ }^{2}$, Osipov Anatoly ${ }^{1}$, Patseev Sergey ${ }^{3}$, Aksiuchyts Alexander ${ }^{1}$, \\ Ponomarenko Gennady ${ }^{4}$ and Kulchitsky Vladimir*2 \\ ${ }^{1}$ Belarusian State University of Informatics and Radioelectronics, Belarus \\ ${ }^{2}$ Institute of Physiology, National Academy of Sciences, Minsk, Belarus \\ ${ }^{3}$ First Minsk City Clinical Hospital, Belarus
}

${ }^{4}$ Federal Scientific Center of Rehabilitation of the Disabled named after G.A. Albrecht of the Ministry of Labour and Social Protection of the Russian Federation, Russia

*Corresponding author: Kulchitsky Vladimir, Institute of Physiology, National Academy of Sciences, Minsk, Belarus

\begin{tabular}{|c|c|}
\hline ARTICLE INFO & ABSTRACT \\
\hline Received: 幽 October 27, 2019 & \multirow{2}{*}{$\begin{array}{l}\text { Paper summarizes results of cold c plasma use in medical devices surface treatment, } \\
\text { focusing at manufacture applications. Attention is given to results of changes in the } \\
\text { hydrophilic properties of glass surfaces, stainless steel, polyamide and latex when } \\
\text { exposed to atmospheric plasma, depending on the time of treatment with cold plasma } \\
\text { and distance to the surface. }\end{array}$} \\
\hline Published: 慧 November 05, 2019 & \\
\hline
\end{tabular}
try, Osipov Anatoly, Patseev Sergey, Aksiuchyts Alexander, Ponomarenko Gennady, Kulchitsky Vladimir. Cold Atmospheric Plasma Treatment of Medical Devices. Biomed J Sci \& Tech Res 22(3)-2019. BJSTR. MS.ID.003766.

Abbreviations: Ar: Argon Gas; CAPP: Cold Atmospheric-Pressure Plasma; CM: Centimeter; MFC: Mass Flow Corrector

\section{Introduction}

Medical devices are used for diagnosis and prevention $[1,2]$, therapy [2,3], rehabilitation of patients, and depending on the purpose, require sterilization in accordance with related regulations [4]. Unfortunately, the sterilization process is associated with some damage to medical devices, depending on its intensity. Thus, the development of effective and gentle sterilization methods for medical devices is important and not yet resolved. The article presents material on the advisable use of cold atmospheric plasma for medical devices processing $[1,4,5]$. Cold atmospheric-pressure plasma (CAPP) is a mixture of neutral and metastable atoms and molecules of gas that provides heating of the object under treatment to a temperature of not more than $40^{\circ} \mathrm{C}$ [1-5]. One of the possible application areas is to modify the surfaces of various materials in order to improve adhesion and hydrophilicity properties. When processing the material under atmospheric pressure plasma, the surface is decontaminated and sterilized. Cleaning is carried out by the interaction of the plasma jet (generated due to the gas-dynamic acceleration of the working gas) with the surface of the material.

In this case, physically adsorbed impurity particles are removed or a chemical reaction with gases occurs with the transfer of reaction products into the gas phase, as well as cleaning by gas-plasma flows is observed. As a result of these processes, it is possible to achieve a significant increase in the wettability of materials of various nature. In addition, during the activation of the surface, free radicals are formed, and a chemically active surface layer is formed. In this paper, we studied the effects of CAPP on materials widely used in healthcare: medical steel (scalpels, clamps, dental instruments, etc.), glass (laboratory glass, dishes, etc.), polyamide material (surgical sutures, prostheses and orthopedic products, etc.), latex (medical gloves, etc.) [1,6,7]. Increasing the hydrophilicity of the surface of medical devices made of the materials under study will significantly improve their performance properties [8]. 


\section{Materials and Methods}

During the study we processed the surface of the K8 glass, AISI-304 stainless steel, polyamide, and latex by cold plasma of atmospheric pressure (Figure 1). The treatment of materials surface was carried out using an experimental set-up, the schematic illustration of which is shown in Figure 1. The set-up consisted of a balloon with

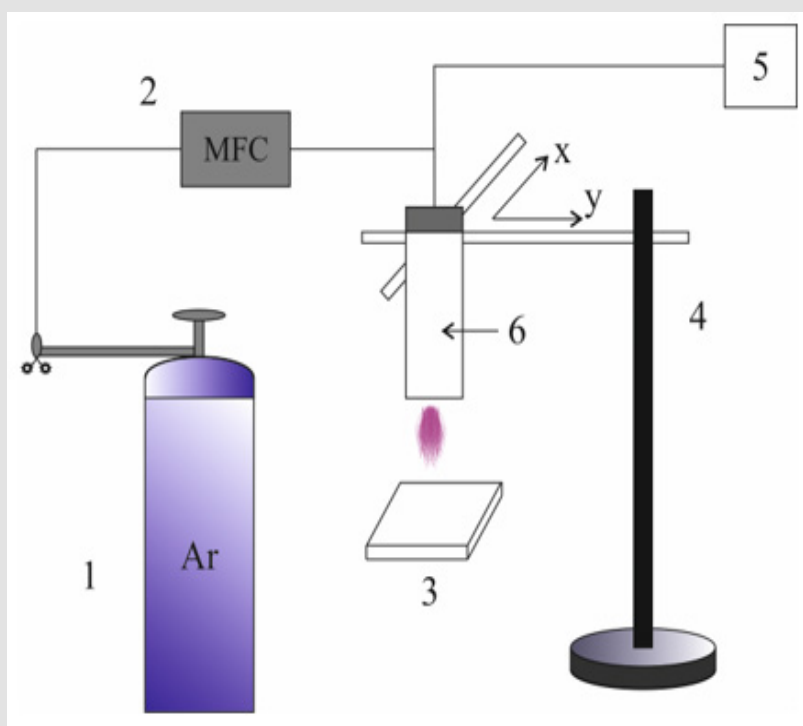

Figure 1: Schematic illustration of the experimental set-up.

(1) Argon gas,

(2) Gas mass flow corrector,

(3) Processing substrate,

(4) Holder,

(5) Power source,

(6) Discharge system.

The set-up was based on the application of a coaxial discharge system with dielectric barrier discharge to create diffuse plasma at atmospheric pressure. Such discharge system enabled generating a plasma jet of $3 \mathrm{~cm}$ length with a processing area of circa $1 \mathrm{~cm}$ diameter. Argon was used to generate the plasma. The surface treatment of glass, stainless steel, polyamide and latex was carried out at a discharge power of 25 Watt and a flow rate of argon of 300 liter per hour. The temperature in the center of the plasma jet, measured by the contact method, was no more than $40^{\circ} \mathrm{C}$. The evaluation of the surface adhesion of the studied materials based on the sessile drop method, which was used to measure the contact angle $\varphi[1,8]$. To determine the contact angle, a drop of about $0.01 \mathrm{ml}$ was applied to the surface, then it was photographed, and the contact angle was calculated using the AutoCAD program. To study the dependence between the processing time and the change of surface materials hydrophilicity $\varphi(t)$, the processing time was increased up to 60 seconds with an interval of $\Delta t$. The processed image of a water drop photograph is shown in Figure 2. To quantify the change in the contact angle, the effective surface treatment time $T_{\text {eff }}$ is calculated by the formula as follows:

$$
T_{e f f} \geq \frac{s}{\varphi_{0}},
$$

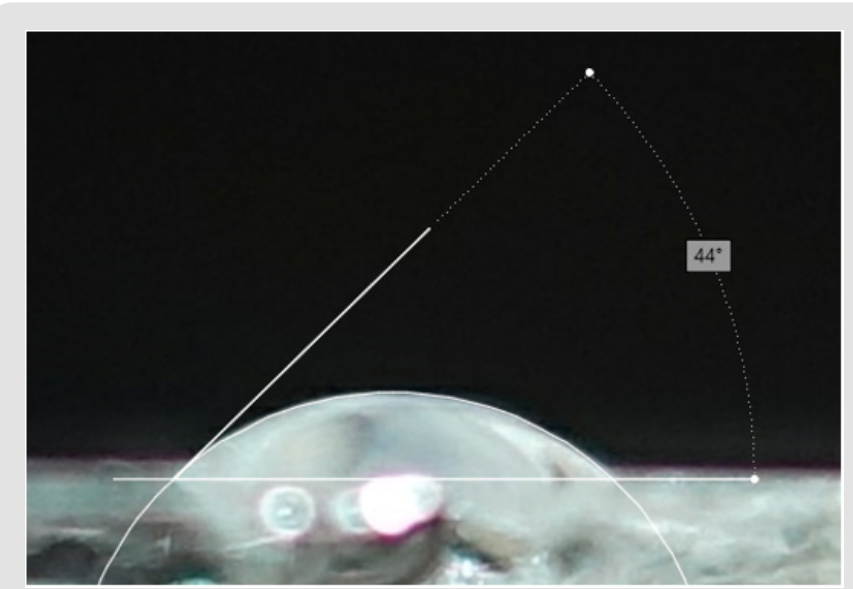

Figure 2: Image of a sessile drop on the glass surface with contact angle measured by AutoCAD.

where $\varphi_{0}$ is the contact angle measured at the beginning of the study $\left(t=0_{\text {sec }}\right)$;

$\mathrm{S}$ is the area bounded by the curve $\varphi(t)$ and y-axis. At regular intervals $\Delta t$, the area $S$ is calculated in accordance with the following formula:

$$
S=\frac{\varphi_{0} \Delta t}{2}+\frac{\varphi_{n} \Delta t}{2}+\sum_{i=1}^{n-1} \varphi_{i} \Delta t
$$

where $i$ is the consecutive number of the contact angle measurement, $i=0 \ldots n$.

\section{Results and Discussion}

Figure 3 illustrates the dependence of the changes in the contact angle on the processing time of steel and glass $\varphi(t)$ at discharge system - substrate distance $1.5 \mathrm{~cm}$ (Figure 3). The presented dependences prove that the adhesive properties of glass improved by almost three times during the first 10 seconds. The effective surface treatment time is $T_{\text {eff }} \geq 17 \mathrm{sec}$. In this period, impurity particles are effectively removed as well as cleaning is made at the atomic level. During further processing (over $17 \mathrm{sec}$.) the rate of surface activation slows down significantly, which results in no significant improvement of adhesion properties. Studies of the hydrophilicity of the treated stainless steel showed the following results. Stainless steel is not a hydrophilic substance, as the contact angle of the untreated surface is $75^{\circ}$. Accordingly, the change in the quantitative indicators of the dependence of the contact angle for steel and for glass on the processing time is different, but the type of dependencies is identical. These dependencies are exponential. Thus, the main effect of the treatment for steel is achieved in time $T_{\text {eff }} \geq 19.7 \mathrm{sec}$. Within this time frame the contact angle has reduced from $75^{\circ}$ to $22^{\circ}$. The processing time between 20 to 30 seconds is sufficient to achieve a minimum contact angle for stainless steel. 


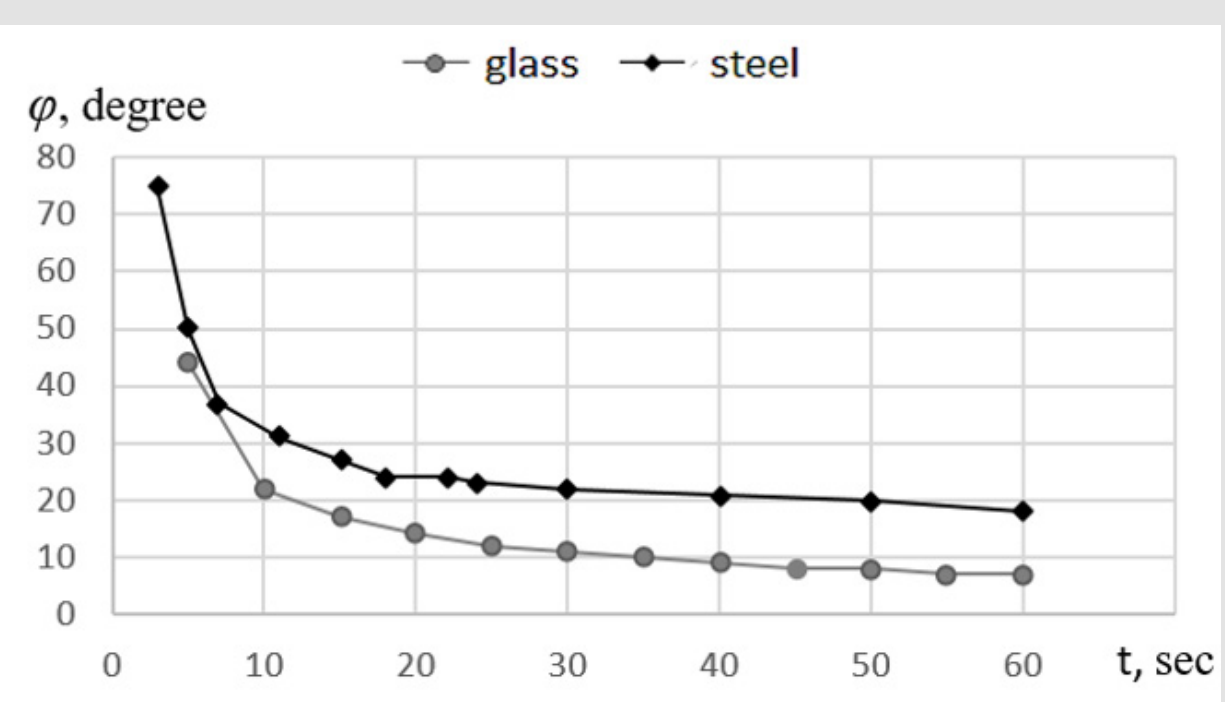

Figure 3: Dependence of the contact angle on the surface of glass and stainless steel on the processing time.

The study of the dependences of the changes in the contact angle of glass and stainless steel on the distance between the tube of the discharge system and the surface being treated was carried out for a processing time of $30 \mathrm{sec}$. Respective dependences $\varphi(t)$ are illustrated in Figure 4. Both dependencies have a pronounced extremum. The minimum contact angle is achieved by the treatment carried out at a distance of $1.5 \mathrm{~cm}$ from the end of the discharge device. At this distance, the drop almost completely spreads on glass (the contact angle is 11 degrees). $22^{\circ}$ is the minimum contact angle for steel. The largest treatment area is achieved at this distance with a plasma jet of $2.2 \mathrm{~cm}$ length. Thus, the increase or decrease of the distance to the treated surface results in the decrease of the efficiency of atmospheric plasma treatment. According to the study, the distance of the effective surface treatment of steel and glass that would lead to its best adhesion lies in the range from 1 to 2 $\mathrm{cm}$. The ratio of the contact angle at the minimum and maximum distance to the contact angle of the extremum $K_{2}=\varphi_{d \min } / \varphi_{\text {dext }}$ and $K_{2}=\varphi_{d \max } / \varphi_{\text {dext }}$ for glass amounts to $K_{1}=5.8$ and $K_{2}=6.8$, as well as for steel it is $K_{1}=1.3$ and $K_{2}=2$ respectively. Thus, compared with steel, glass is more sensitive to the choice of the distance from the Jet to the surface under treatment (Figure 4).

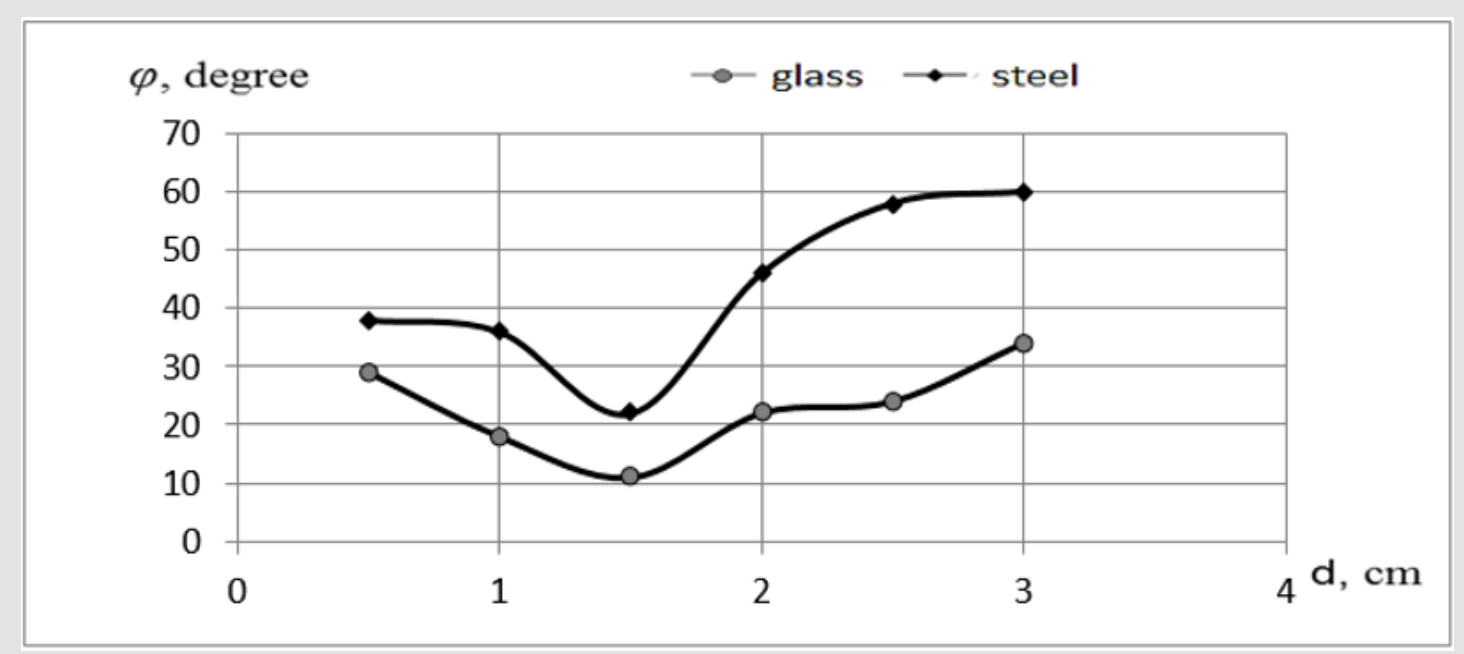

Figure 4: Dependence of the changes in the contact angle of glass and stainless steel on the distance 'discharge system substrate'.

Figure 5 shows the dependence of the contact angle on the surface processing time for polyamide and latex materials at the distance discharge system - substrate $1.5 \mathrm{~cm}$ (Figure 5). The study of the effect of atmospheric plasma on latex for 1 minute showed that the contact angle of the latex surface changed from $96^{\circ}$ to $35^{\circ}$, and the time of effective surface treatment was $T_{\text {eff }}=29 \mathrm{sec}$. During the processing of polyamide, the contact angle was decreasing from $67^{\circ}$ to $12^{\circ}$ during the first 50 seconds, then in the time frame from 
50 seconds to 60 seconds, it was increasing. This indicates that the structure (morphology) of the material on the surface is being changed. Thus, the processing time of the polyamide material to increase the adhesive properties should not exceed a certain period of time during which the surface structure does not change (for the selected sample of polyamide it is no more than 50 seconds). The effective processing time for polyamide is $T_{\text {eff }}=20.5 \mathrm{sec}$. Graphs of the dependence of the distance from the end of the discharge device on the contact angle of polyimide and latex at $30 \mathrm{sec}$ plasma treatment are shown in Figure 6. The presented graphs show that the maximum wettability is achieved at the minimum distance from the end of the discharge device to the surface at which the studies were performed (for latex it is $0.5 \mathrm{~cm}$, for polyamide it is $1 \mathrm{~cm}$ ). The presented dependencies do not have any extrema similar to steel or glass and are monotonously increasing.

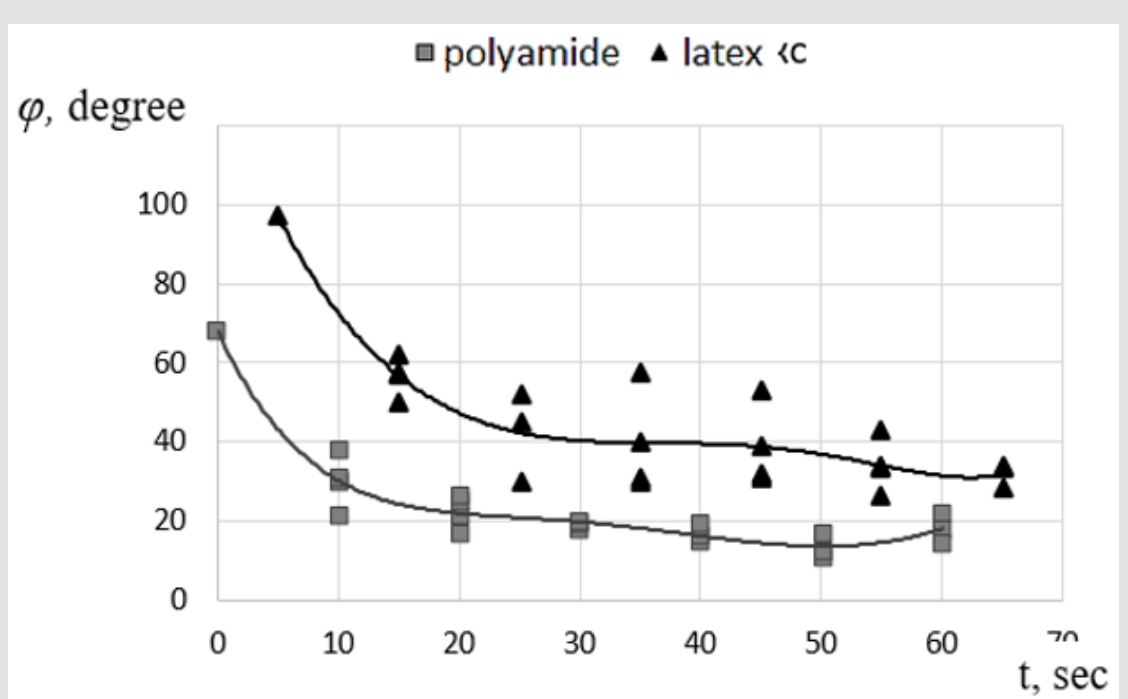

Figure 5: Dependence of the contact angle of the polyamide and latex surface on the processing time.

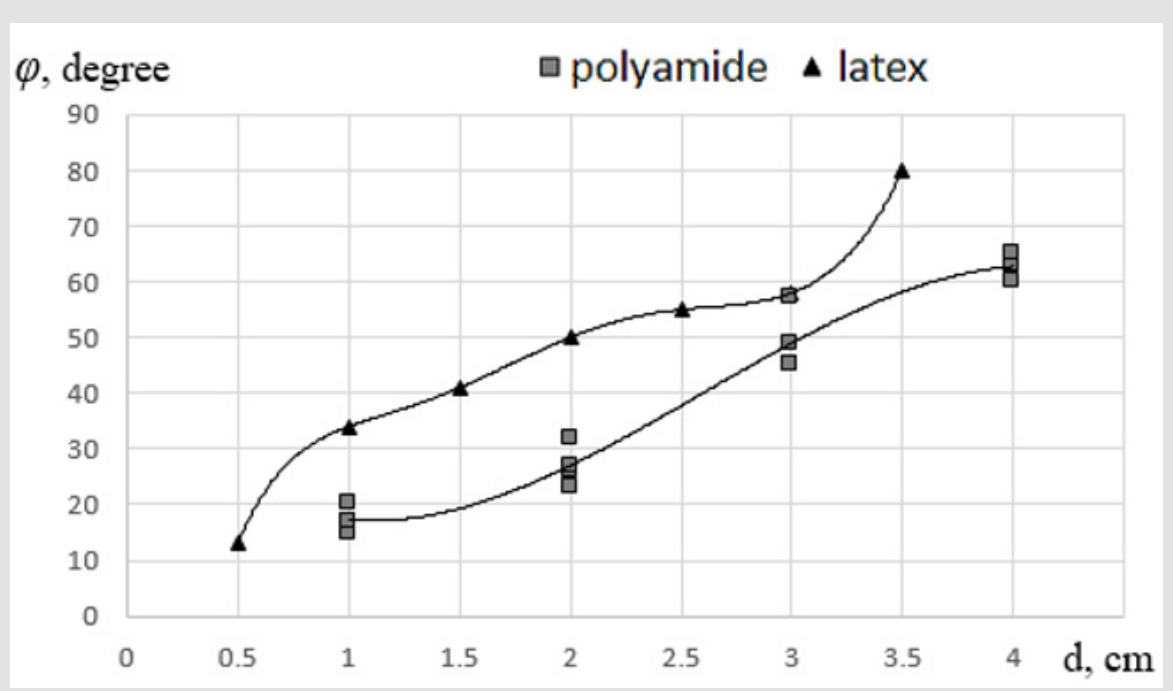

Figure 6: Dependence of the contact angle of polyimide and latex on the distance 'discharge device - substrate'.

\section{Conclusion}

The following results were obtained based on the study conducted.

1. It was confirmed that atmospheric plasma changed the surface properties of various materials: glass, metal, polyamide, and latex. Accordingly, atmospheric plasma was a universal means of changing the properties of surfaces, since it affected materials of various nature. The effect of reducing the contact angle was to remove the impurity and clean the surface at the atomic level.

2. The visible length of the jet in the study was $2.5 \mathrm{~cm}$. The best results in improving the adhesive properties for steel and glass were obtained at the distance of $1.5 \mathrm{~cm}$ from the end of the discharge device to the surface under treatment. For polyamide 
and latex, the best effect was obtained with minimum distances of $0.5 \mathrm{~cm}$ and $1.0 \mathrm{~cm}$, respectively, from the end of the discharge device to the treated surface. This indicates that the effective area of plasma jet exposure for these materials does not exceed its visible part.

3. A significant improvement in the adhesive surface properties was achieved during a one-minute treatment under atmospheric discharge plasma: for glass, the contact angle decreased from $44^{\circ}$ to $5^{\circ}$ (almost complete spreading), for stainless steel - from $75^{\circ}$ to $18^{\circ}$, for latex - from $96^{\circ}$ to $35^{\circ}$, and for polyamide - from $67^{\circ}$ to $12^{\circ}$. The best result among the studied materials was obtained for glass, its contact angle decreased by 8 times.

4. The dependence of the contact angle on the time of treatment by atmospheric plasma is exponential and significantly decreases while the treatment time increases. The effective processing time T_eff amounts to above $19.7 \mathrm{sec}$. for steel, above $17 \mathrm{sec}$. for glass, above $20.5 \mathrm{sec}$. for polyamide, and above 29 sec. for latex. For polyamide, an increase in the contact angle is observed at the end of the processing time apparently due to the change in the film structure of the material. Therefore, the choice of the processing time should be determined based on the processing efficiency criterion, but not more than a certain maximum period.

Thus, treatment of materials by cold atmospheric pressure plasma is a promising technology for the production of medical products, as it leads to the improvement of their performance properties by increasing the surface adhesion of materials of various nature. At the next stage of research, microbiological studies will be carried out to clarify the effectiveness of the disinfecting effect of cold plasma. To this end, microbiologists and specialists in the field of sanitary hygiene will be involved. It is also planned to experimentally adjust required time intervals exposure, the optimal duration and intensity of exposure, as well as the series of procedures.

\section{Conflict of Interest}

All listed authors concur with the submission of the manuscript; all authors have approved the final version. The authors have no financial or personal conflicts of interest.

\section{References}

1. Isbary G, Shimizu T, Li YF, Stolz W, Thomas HM, et al. (2013) Cold atmospheric plasma devices for medical issues. Expert Rev Med Devices 10(3): 367-377.

2. Bernhardt T, Semmler ML, Schäfer $M$, Bekeschus $S$, Emmert $S$, et al. (2019) Plasma Medicine: Applications of Cold Atmospheric Pressure Plasma in Dermatology. Oxid Med Cell Longev pp. 3873928.

3. Bauer G, Sersenova D, Graves DB, Machala Z (2019) Cold Atmospheric Plasma and Plasma-Activated Medium Trigger RONS-Based Tumor Cell Apoptosis. Sci Rep 9: 14210.

4. Jiao Y, Tay FR, Niu LN, Chen JH (2019) Advancing antimicrobial strategies for managing oral biofilm infections. Int J Oral Sci 11: 28.

5. VON Woedtke T, Schmidt A, Bekeschus S, Wende K, Weltmann KD (2019) Plasma Medicine: A Field of Applied Redox Biology. In Vivo 33(4): 10111026

6. Kulchitsky V, Zamaro A, Shanko Y, Koulchitsky S (2018) Positive and negative aspects of cell technologies in cerebral diseases. J Neurol Stroke 8(2): 87-88

7. Meghdadi M, Atyabi SM, Pezeshki Modaress M, Irani S, Noormohammadi Z, et al. (2019) Cold atmospheric plasma as a promising approach for gelatin immobilization on poly( $\varepsilon$-caprolactone) electrospun scaffolds. Prog Biomater 8(2): 65-75.

8. Boyce JM (2016) Modern technologies for improving cleaning and disinfection of environmental surfaces in hospitals. Antimicrob Resist Infect Control 5: 10 .
ISSN: 2574-1241

DOI: 10.26717/BJSTR.2019.22.003766

Kulchitsky Vladimir. Biomed J Sci \& Tech Res

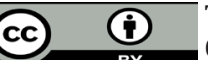

This work is licensed under Creative Commons Attribution 4.0 License

Submission Link: https://biomedres.us/submit-manuscript.php

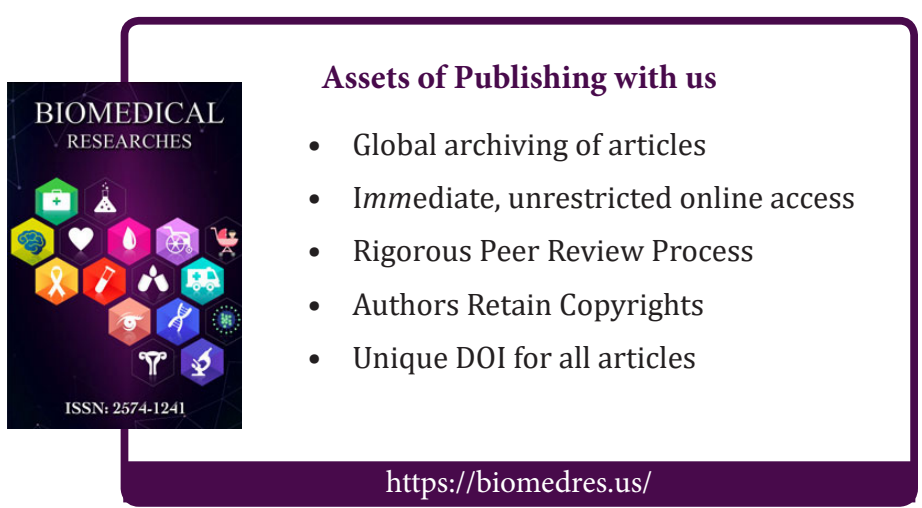

